\title{
A protective hinge wire, intersecting the osteotomy plane, can reduce the occurrence of perioperative hinge fractures in medial opening wedge osteotomy
}

\author{
Firat Gulagaci ${ }^{1,3,4} \cdot$ Christophe Jacquet $^{1,3,4} \cdot$ Matthieu Ehlinger $^{2} \cdot$ Akash Sharma $^{1,3,4} \cdot$ Kristian Kley $^{1,3,4}$. \\ Adrian Wilson ${ }^{5} \cdot$ Sebastien Parratte ${ }^{1,3,4} \cdot$ Matthieu Ollivier ${ }^{1,3,4}$
}

\begin{abstract}
Purpose A recent study reported that positioning a K-wire to intersect the cutting plane at the theoretical lateral hinge location increases the lateral hinge's resistance to fracture during the opening of opening wedge high tibial osteotomy (OWHTO). The purpose of this study was to evaluate the clinical relevance of the use of this K-wire and its benefits in terms of lateral hinge protection during OWHTO in daily practice.

Methods A retrospective comparative study identified 206 patients who underwent OWHTO from January 2014 to December 2017. Among these patients, 71 had an additional K-wire (HK + group), whereas 135 did not (HK- group). The subjects meeting the inclusion criteria were included in a matched pairing process, which identified 60 patients in the $\mathrm{HK}+$ group and 60 patients in the $\mathrm{HK}$ - group. Mean follow-up time was $2.3 \pm 1.0$ years (range 2-4.2). Radiographic outcomes were evaluated with intraoperative and postoperative fluoroscopic imaging and with CT imaging at 6 weeks post OWHTO surgery. The knee osteoarthritis outcomes score (KOOS) was used and time needed to return to work and any kind of sports was collected. Results Thirty six patients (30\%) were found to have a LHF. Among these patients, 26 (72\%) did not have an additional K-wire positioned at their theoretical lateral hinge location (HK-group) during the procedure. LHF rate for patients without additional $\mathrm{K}$-wire group $(\mathrm{HK}-)$ was $43.3 \%$, whereas it was $16.7 \%$ for the patients with an additional $\mathrm{K}$-wire $(\mathrm{HK}+)$ [Odd ratio $3.895 \% \mathrm{CI} 1.6-8.3 ; p=0.005$ ]. The mean time to return to work, return to any kind of sports, and bone union was significantly shorter for $\mathrm{HK}+$ group $(p<0.05)$.

Conclusion This study demonstrated that during OWHTO, positioning a K-wire intersecting the cutting plane at the theoretical lateral hinge location reduced the number of intraoperative lateral hinge fractures.

Level of evidence III retrospective case-control study.
\end{abstract}

Keywords HTO $\cdot$ Hinge fracture $\cdot$ Postoperative outcomes $\cdot$ Patient specific cutting guide

Matthieu Ollivier

matthieu.ollivier@ap-hm.fr

1 Institute of Movement and Locomotion Department of Orthopedics and Traumatology, St Marguerite Hospital, 270 Boulevard Sainte Marguerite, BP 29, 13274 Marseille, France

2 Service de Chirurgie Orthopédique et de Traumatologie, CHU Hautepierre, Hôpital de Hautepierre, Hôpitaux Universitaires de Strasbourg, 1 Avenue Molière, 67098 Strasbourg Cedex, France
3 APHM, CNRS, ISM, Sainte-Marguerite Hospital, Institute for Locomotion, Department of Orthopedics and Traumatology, Aix Marseille University, Marseille, France

4 The Institute for Locomotion, Aix-Marseille University, Marseille, France

5 The Wellington Hospital, Wellington Place, St. John's Wood, London, UK 


\section{Introduction}

Osteotomy around the knee is well established as an effective treatment for offloading an osteoarthritic compartment, where there is an unfavorable alignment. There are options of opening or closing techniques in the distal femur and proximal tibia [1-3]. The predominant technique is the medial opening wedge high tibial osteotomy and the most common indication is medial compartment osteoarthritis [4-8].

Over the last two decades, the outcomes have improved in osteotomy around the knee by redefining the indications, improving preoperative planning and by making the surgical technique more accurate, safer, and reproducible.

With preoperative and intraoperative planning to define the hinge point for the osteotomy, whether it is an opening or closing wedge technique, it is important to preserve and maintain the hinge, as this is critical to facilitate bone healing and to provide stability to the osteotomy $[9,10]$. If the hinge is compromised, the fixation may not be sufficient and secondary loss of correction may occur [11].

Dexel et al. [9] reported an incidence of $30.4 \%$ for lateral tibial hinge fractures related to opening wedge high tibial osteotomy (OWHTO), half of these occurred during the procedure and the other half after 6 weeks of follow up. Therefore, it can be assumed that finding a solution to preserve the hinge intraoperatively is crucial for an optimal outcome following OWHTO.

Several techniques that have been developed to minimize hinge point issues such as taking care with the saw depth cut and subsequent speed with which the wedge is then opened. Other authors have developed protective cutting systems $[12,13]$ and used drilling techniques to protect the hinge by decreasing the stress at the hinge point by placing an anteroposterior protecting hinge point wire $[14,15]$.

The use of patient-specific cutting guides [2] (PSCGs) requiring preoperative $\mathrm{CT}$ scan templating during OWHTO has raised the possibility of making instrumentation specific to each patient. Using these guides, a cadaveric study [11] reported that positioning a K-wire to intersect the cutting plane at the theoretical lateral hinge location increases the lateral hinge's resistance to fracture during the opening.

The aim of this study was to evaluate the clinical relevance of the use of this K-wire and its benefits in terms of lateral hinge protection during OWHTO with PSCGs in daily practice.

We hypothesized that the addition of an intersecting $\mathrm{K}$-wire decreases the incidence of intraoperative hinge fractures during OWHTO and augments bone healing, thus reducing lateral hinge fracture rate in the early phases of rehabilitation.

\section{Materials and methods}

After gaining local ethics committee's approval, a retrospective comparative study identified 206 patients who underwent OWHTO from January 2014 to December 2017. Among these patients, 71 had an additional K-wire positioned at their theoretical lateral hinge location (HK + group), whereas 135 did not (HK- group). All the surgeries were performed by two different surgeons who had practiced more than 20 OWHTO using PSCGs before January 2014 to avoid biases related to learning curve effect [16]. Between January 2014 and June 2016, no K-wire was used during the surgery. After June 2016, the K-wire was systematically used according to the experimental results of biomechanical cadaveric study [11].

Mean follow-up time was $2.3 \pm 1.0$ years (range 2-4.2).

Inclusion criteria were: patients $<65$ years of age with isolated medial knee osteoarthritis (Ahlbäck $\leq 2 / /$ Kellgren and Lawrence $<4)[17,18]$ and significant metaphyseal tibial vara (medial plateau tibial angle MPTA $<85^{\circ}$ ).

Exclusion criteria were: advanced medial knee osteoarthritis (Ahlbäck >2) evidence of symptomatic patellofemoral or lateral knee osteoarthritis, previous ipsilateral knee surgery (except meniscus related arthroscopic procedures), history of fragility fracture, evidence of osteopenia or osteoporosis, post-traumatic arthritis. Patients requiring simultaneous anterior cruciate ligament reconstruction, meniscus, or cartilage surgery were also excluded.

Patient consent was collected preoperatively after they were informed of the procedure in accordance with the principles of the Helsinki Declaration. For patients meeting the inclusion criteria, a matched pairs process identified 60 patients in the $\mathrm{HK}+$ group and selected 60 patients from the HK- group, matching criterion were age ( \pm 3 years) and preoperative MPTA value $( \pm 2$ degrees reflecting the extent of correction to be performed).

The mean age of patients of $\mathrm{HK}+$ and $\mathrm{HK}-$ groups was $44.9 \pm 7.7$ and $45.8 \pm 4.3$ ( $p=$ NS), respectively. The groups were comparable in terms of demographic background (and radiological parameters (Table 1).

In the preoperative planning stage, the planned correction was first calculated by the surgeon using conventional radiographs (weight-bearing long leg, A/P, and lateral views) following Miniaci's method [19]. Subsequently, all patients underwent a CT scan and patient-specific cutting guides (PSCG) osteotomy following a previously published surgeon guided step-by-step procedure [2] Fig. 1.

The lateral cortex was protected in the $\mathrm{HK}+$ group using a $\varnothing 2.2 \mathrm{~mm} \mathrm{~K}$-wire. It was placed from distal-toproximal from anteromedial-to-lateral to intersect the cutting plane at the theoretical hinge point $(10 \mathrm{~mm}$ from the lateral cortex). Subsequently, the osteotomy cut was 


\begin{tabular}{llll}
\hline Parameters & HK +group $(n=60)$ & HK- group $(n=60)$ & $p$ value \\
\hline Age & $44.9 \pm 7.7$ & $45.8 \pm 4.3$ & 0.4 \\
Gender (male) $(\%)$ & $32(53.3)$ & $35(58.3)$ & 0.3 \\
Active smoking $(n)$ & 6 & 4 & 0.7 \\
Preoperative MPTA $\left(^{\circ}\right)$ & $82 \pm 4$ & $81 \pm 4$ & 0.4 \\
Planned correction MPTA $\left(^{\circ}\right)$ & $9 \pm 5$ & $8 \pm 4$ & 0.5 \\
Operative time (mins) & $26 \pm 10$ & $26 \pm 11$ & 0.8 \\
Fluoroscopic image $(n)$ & $5.1 \pm 3.2$ & $5.7 \pm 4.5$ & 0.4 \\
Osteotomy's gap grafting (allograft/CPC) & $19 / 31$ & $17 / 33$ & 0.6 \\
Postoperative MPTA $\left(^{\circ}\right)$ & $90 \pm 4$ & $90 \pm 3$ & 0.4 \\
Follow up (years) & $2.2 \pm 0.9$ & $2.4 \pm 1.0$ & 0.1 \\
\hline
\end{tabular}

$H K \mathrm{~K}$-wire positioned in theoretical lateral hinge location, MPTA medial proximal tibial angle, $C P C$ calcium phosphate cement

$P$ significant if $<0.05$
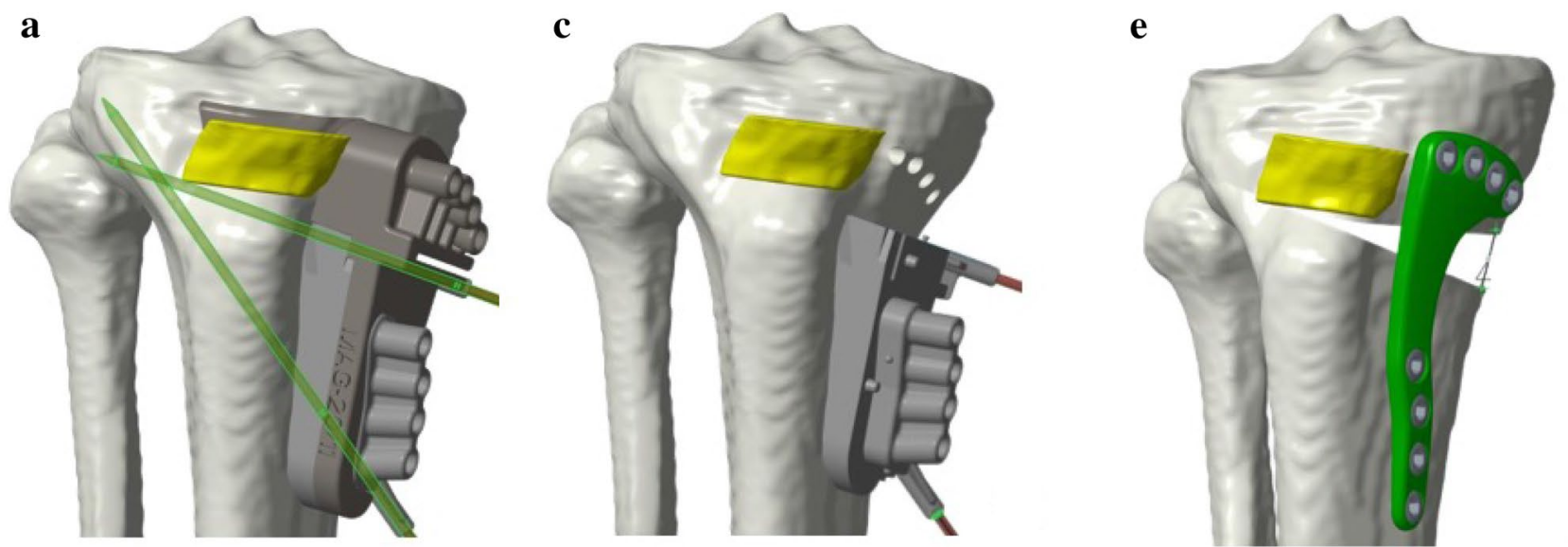

b
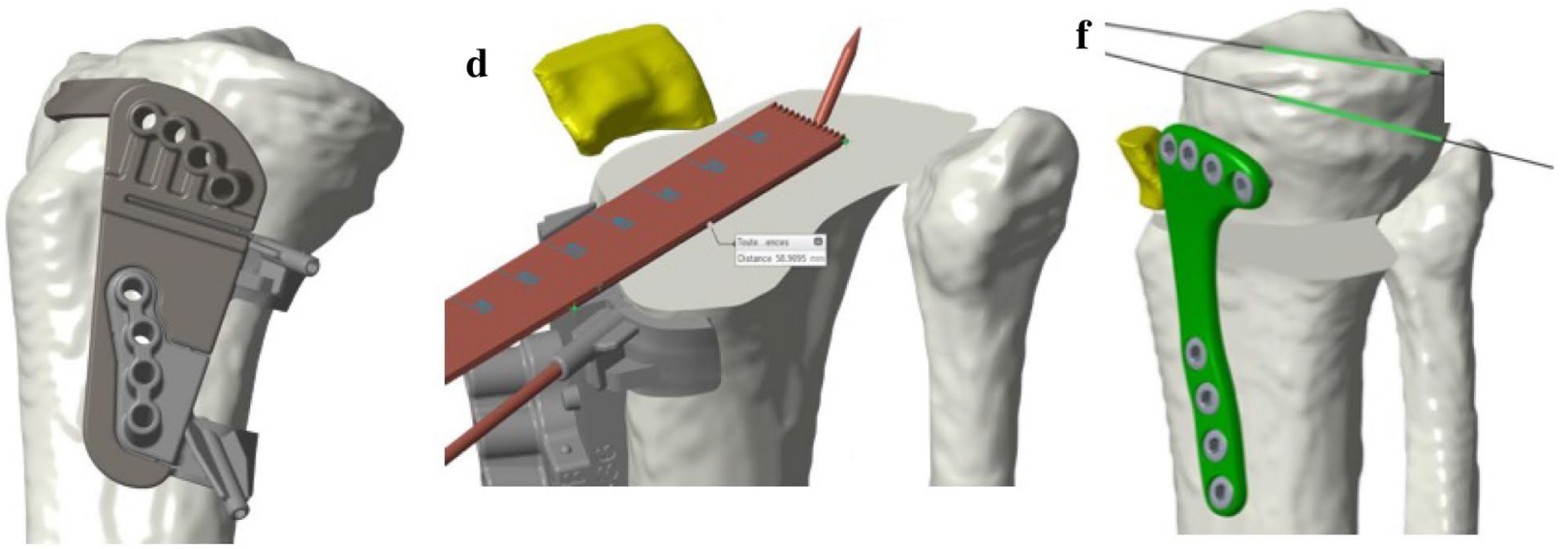

Fig. 1 Preoperative 3D templating illustrating the operative technique of custom cutting guides for the HK + group. a, b Optimal positioning of the cutting guide with two K-wires (one to protect the lateral hinge during the osteotomy ( $\mathrm{HK}+$ group) and one to guide the saw))/ c pre-drilling the eight holes of the plate with the guide/ d illustration of lateral hinge protection by K-wire/ e, f opening of the osteotomy with definitive positioning of the plate and screws on the pre-drilled holes 
performed and was gradually opened/distracted with a laminar spreader until the pre-drilled screw holes were aligned with the holes in the plate. The plate was secured using eight screws, the size of which were pre-determined during preoperative planning.

The bone defect was filled with femoral head wedge allograft or injectable calcium phosphate cement (Quickset, Graftys ${ }^{\circledR}$, Aix-en-Provence, France). Figures 2 and 3 illustrate an example of hinge's protection obtained using the intersecting K-wire for a $11 \mathrm{~mm} / 12^{\circ}$ opening.

\section{Patients' evaluation and follow up}

All lateral hinge fractures (LHF) were evaluated according to the Takeuchi classification [3].

Patients were evaluated prospectively at postoperative first, third, and sixth months, and then every 6 months. Radiographic outcomes were evaluated with intraoperative and postoperative fluoroscopic imaging and with $\mathrm{CT}$ imaging at 6 weeks post OWHTO surgery. Every radiographs or CT scans were reviewed by two senior clinicians (one radiologist and one surgeon), in cases of different interpretation,

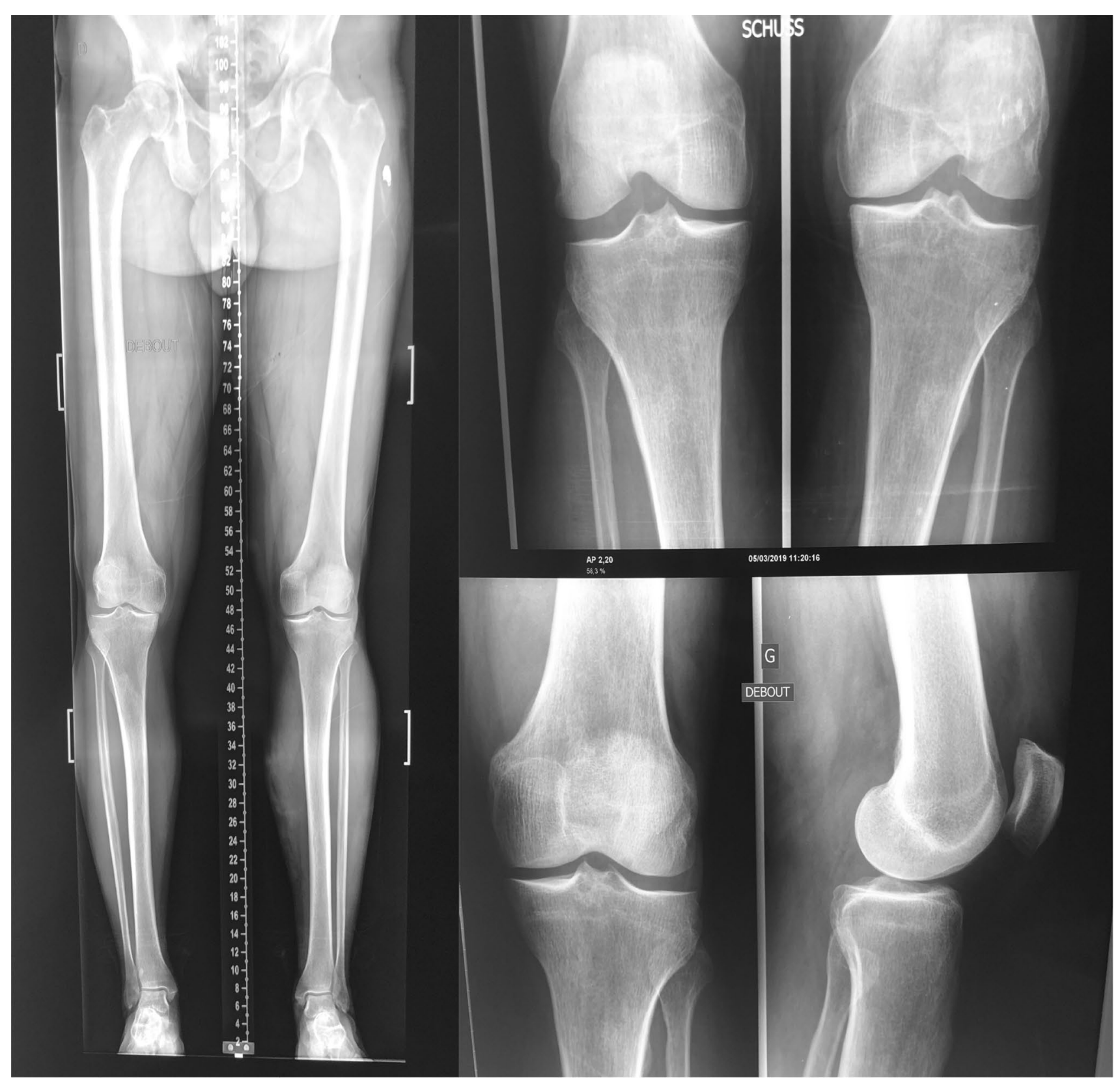

Fig. 2 51-years-old patient suffering from painful right knee for more than 2 years. Preoperative examination exhibits an important metaphyseal tibial varus with Alback stage 2 arthritis on schuss X-rays ( $30^{\circ}$ flexion PA weight-bearing) 
Fig. 3 Hinge K-wire protecting a $11 \mathrm{~mm} / 12^{\circ}$ medial opening before and after allografting the osteotomy's bone gap

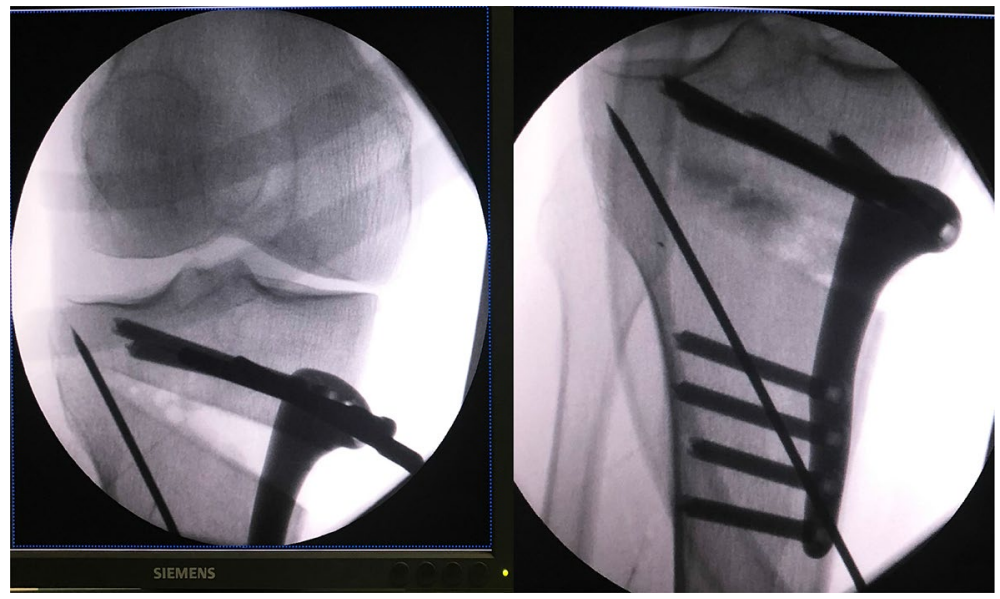

a third evaluation was performed by the two clinicians to reach a definitive interpretation. The Kappa coefficient of the first evaluation was 0.95 . The timing of LHF was defined as intraoperative fractures; diagnosed either during the procedure or immediately postoperatively with a plain radiograph (same day) or postoperative fractures diagnosed on CT images (not viewed on radiographs) at 6 weeks or fractures diagnosed on radiographs performed during subsequent follow up. Figure 4 exhibits CT examination of the hinge integrity.

The same postoperative regime was used in both the groups, including:

- Full weight-bearing if no LHF occurred intraoperatively.
- For Takeuchi types 1 and 2 diagnosed intraoperatively (Fig. 5); toe touch weight-bearing for 6 weeks aided with the use of crutches was recommended. Full weight-bearing was allowed after 6 weeks.

- For Takeuchi type III diagnosed intraoperatively: the fracture was treated intraoperatively by two additional compression screws (Fig. 6) and no weight-bearing during 6 weeks followed by toe touch weight-bearing for 6 weeks aided with the use of crutches was recommended. Full weight-bearing was after allowed 12 weeks.

- If LHF was diagnosed at 6 weeks' follow up, for the types I and 2, full weight-bearing was extended. For the type III a toe touch weight-bearing for 6 weeks was recommended before full weight-bearing.

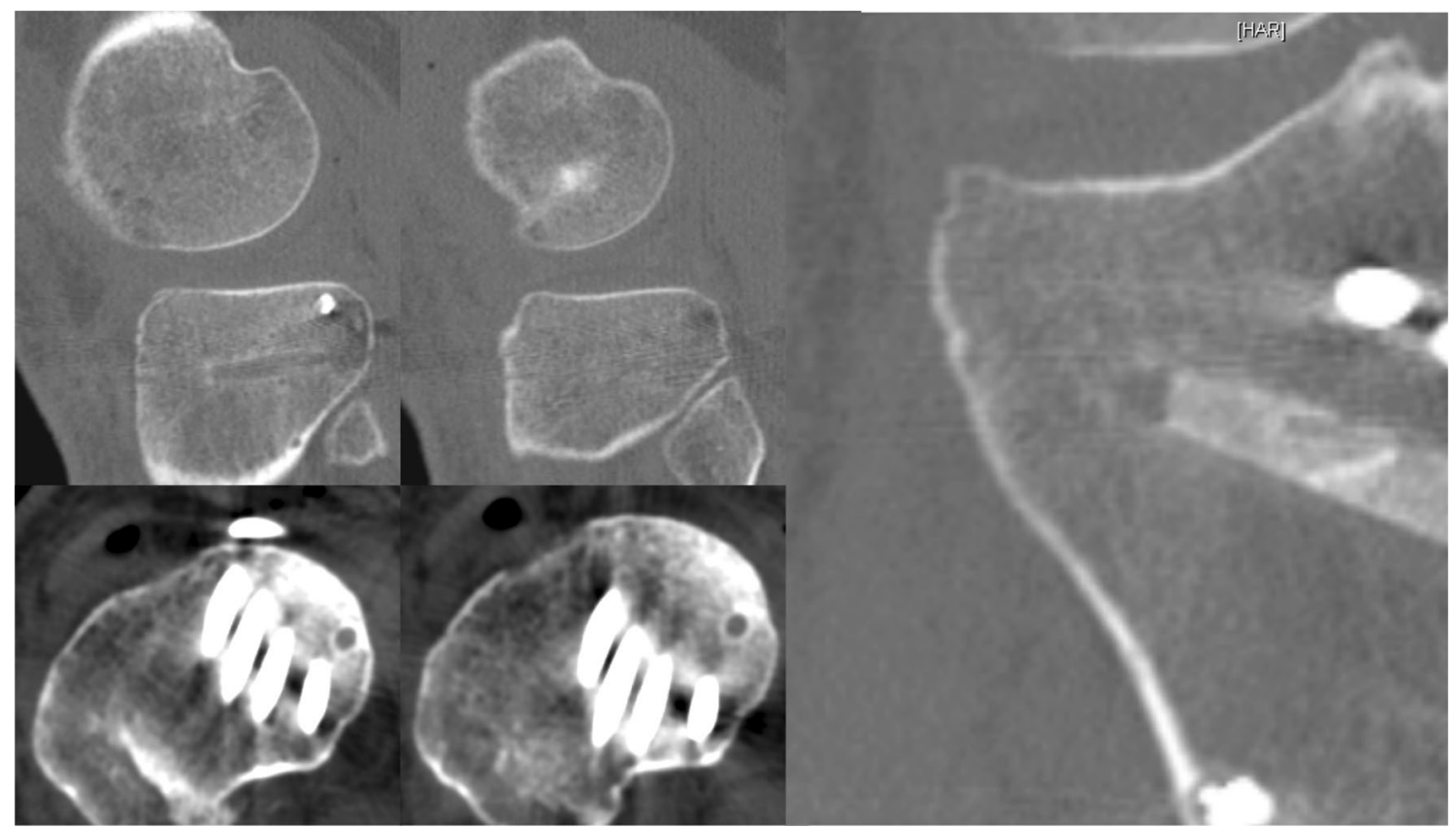

Fig. 4 CT scan examination at 4-6 weeks focusing on potential hinge fracture, no evidence of bone fracture was found in this case 


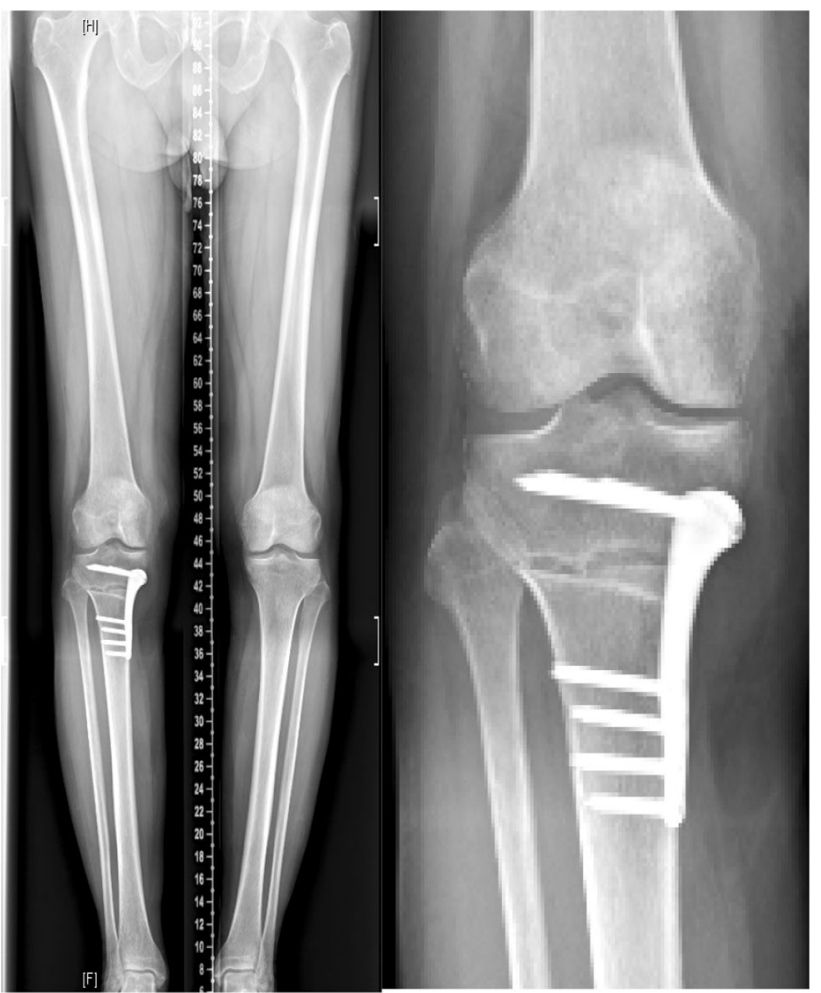

Fig. 5 Example of a Takeuchi type 2 hinge fracture diagnosed postoperatively

Clinical union was defined as painless full weight-bearing and radiological union with the presence of bridging callous of two cortices visible on radiographs [20]. Medial proximal tibial angle (MPTA), posterior proximal tibial angle (PPTA) and hip-knee-ankle angle (HKA) were assessed at the last available follow up. The knee osteoarthritis outcomes score (KOOS) $[21,22]$ was used to identify any complications and to evaluate function and rehabilitation. Patients' return sports and work time were also duly noted.

\section{Statistical analysis}

The descriptive statistics were presented as mean \pm standard deviation for quantitative variables. Mann-Whitney $U$ test for two independent samples with $95 \%$ confidence interval (95\% CI) was used to evaluate the differences between two variables, one-way ANOVA between more than two variables and multiple linear regressions for relationships. Paired student $t$ test was used to estimate evolution of functional outcomes during follow up. The differences in proportions between two samples were estimated with z-test with $95 \%$ CI. Finally, a multivariate analysis evaluating factors related to clinical outcomes was performed. A post hoc power analysis demonstrated that 60 patients by group allow to demonstrate a twofold difference in term of fracture rate in the first six postoperative weeks (expected rate 30\%). PASW statistics version 20 (SPSS, IBM Inc., Chicago, Illinois) was used for statistical analysis. The threshold for statistical significance was set at $p<0.05$.

\section{Results}

Thirty six patients $(30 \%)$ were found to have a LHF. Among these patients, 26 (72\%) did not have an additional $\mathrm{K}$-wire positioned at their theoretical lateral hinge location (HK - group) during the procedure. LHF rate for patients without additional $\mathrm{K}$-wire group ( $\mathrm{HK}-$ ) was $43.3 \%$, whereas it was $16.7 \%$ for the patients with an additional $\mathrm{K}$-wire $(\mathrm{HK}+)(\mathrm{OR} 3.8,95 \%$ CI 1.6-8.3; $p=0.005)$ (Table 2).

According to the Takeuchi lateral hinge fractures classification [3], 30 patients had type 1 LHF (83.3\%), whereas three had type 2 (8.3\%) and remaining three (8.3\%) had type 3 (Table 2).

Among the 30 patients who had type 1 LHF, 23 (77\%) did not have additional K-wire positioned at their theoretical lateral hinge location (HK- group). LHF types according to HK groups were summarized in Table 2.

Among all the 36 LHF, 13 (36.1\%) were observed intraoperatively or on immediate postoperative imaging, 20 (55.6\%) were diagnosed at the 6 weeks follow-up appointment with a CT scan, and three (8.3\%) on further subsequent radiological analysis.

The number of recognized intraoperative fractures was higher in the HK- group $(n=11)$, (OR 6.5, 95\% CI 1.4-30.7) as compared to $\mathrm{HK}+$ patients $(n=2)$.

The number of fractures detected at 6 weeks with CTbased imaging was higher in the HK- group $(n=14)$, (OR $2.7,95 \%$ CI $1-10)$ as compared to HK + patients $(n=6)$.

There was no difference with regards to the number of fractures diagnosed during subsequent follow up ( $p=\mathrm{NS}$ ).

Patients' operative details and radiographic evaluation regarding the difference between planned and final measures $(\Delta)$ are summarized in Table 3.

The mean consolidation period of the osteotomy and the mean time to return to work was significantly longer for patients with type 2 or type 3 LHF ( $p<0.0001$ for both). The mean time to return to any kind of sports was significantly longer for patients with type 3 LHF $(p<0.0001)$. KOOS parameters were not statistically different between LHF groups. The relationship between patient outcomes and LHF types is summarized in Table 4.

Age, gender, mean operative time, number of fluoroscopic images taken during surgery, and mean follow-up times were similar between groups. The mean time to return to work, return to any kind of sports and bone union was significantly shorter for HK + group: mean time to return to work was $2.20 \pm 1.1$ months for HK + group and $2.95 \pm 2.6$ months for HK- group $(p=0.04)$; mean time to return to any 


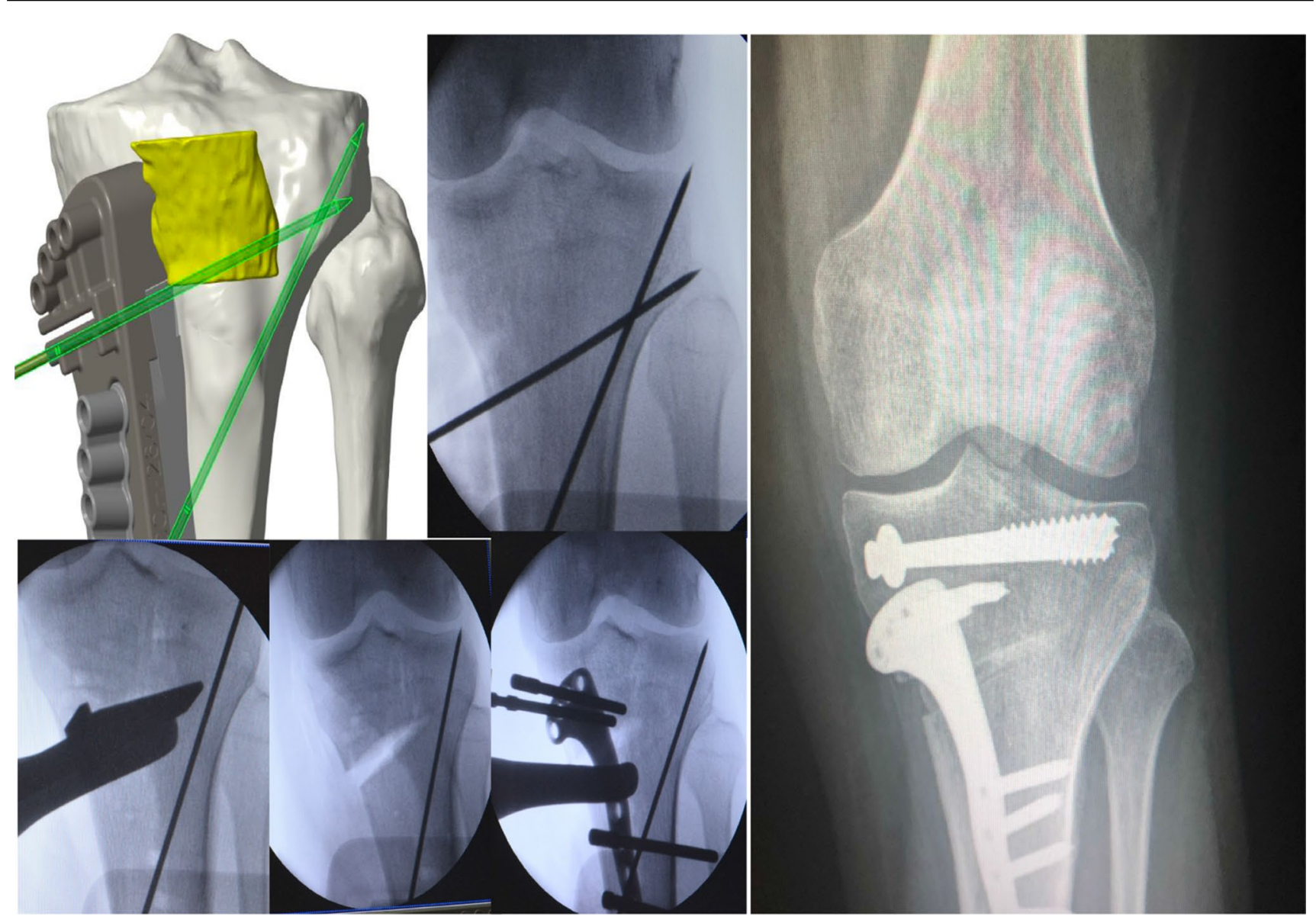

Fig. 6 Example of a Takeuchi type 3 hinge fracture diagnosed and treated intraoperatively

Table 2 LHF types according to HK

\begin{tabular}{lllll}
\hline HK/LHF & No LHF & Type 1 & Type 2 & Type 3 \\
\hline HK + group & $50(41.3 \%)$ & $7(5.8 \%)$ & $1(0.9 \%)$ & $2(1.6 \%)$ \\
HK- group & $34(28.1 \%)$ & $23(19.8 \%)$ & $2(1.6 \%)$ & $1(0.9 \%)$ \\
$p$ & 0.005 & 0.005 & 0.7 & 0.2 \\
Odd ratio (95\% CI) & $6.1(2.6-14.3)$ & $5(2-13)$ & NA & NA \\
Total $(n)$ & $84(69.4 \%)$ & $31(25.6 \%)$ & $3(2.5 \%)$ & $3(2.5 \%)$ \\
\hline
\end{tabular}

$H K \mathrm{~K}$-wire positioned in theoretical lateral Hinge location, LHF lateral Hinge fracture

kind of sports was $4.6 \pm 1.4$ months for $\mathrm{HK}+$ group and $5.9 \pm 4.0$ months for HK - group $(p=0.02)$; mean time for bone union on radiographs was $4.1 \pm 0.9$ months for HK + group and $4.9 \pm 2.6$ months for HK - group $(p=0.02)$ (Table 3).

\section{Other complications}

One nonunion was seen at 9 months after HTO in the $\mathrm{HK}+$ group, union was achieved 5 months after a revision procedure. A total of 11 hematomas were detected postoperatively (seven in HK- group, four in HK + group; $p=\mathrm{NS}$ ). Allograft osteolysis was seen in two patients in HK- group and in one patient in $\mathrm{HK}+$ group ( $p=\mathrm{NS})$. In HK + group, three patients presented with surgical site infections (two superficial infections requiring antibiotic therapy and one deep infection treated by debridement) versus none in the HK- group $(p=0.3)$.

\section{Discussion}

The main finding of this study was that during OWHTO surgery positioning, a K-wire intersecting the cutting plane at the theoretical lateral hinge location reduces the rate of hinge fractures that are detectable in the first 6 weeks postoperatively.

The role of an intact competent lateral tibial hinge in OWHTO has been widely reported to be crucial in its importance for consolidation of the osteotomy and maintenance of angular correction in all three planes [23, 24]. 
Table 3 Patient information according to LHF types

\begin{tabular}{lcccc}
\hline Parameters/LHF type & No LHF $(n=84)$ & Type 1 $(n=30)$ & Type 2 $(n=3)$ & Type 3 $(n=3)$ \\
\hline Operation time (mins) & $25.3 \pm 1.0$ & $22.6 \pm 1.6$ & $55.7 \pm 5.2^{* *}$ & $44.7 \pm 5.2^{* *}$ \\
Fluoroscopic image $(n)$ & $4.5 \pm 0.2$ & $5.2 \pm 0.3$ & $20.7 \pm 1.1^{* *}$ & $19.7 \pm 1.1^{* *}$ \\
$\Delta$ HKA & $1.4 \pm 0.1$ & $1.7 \pm 0.2$ & $1.2 \pm 0.5$ & $1.2 \pm 0.5$ \\
$\Delta$ MPTA & $0.5 \pm 0.1$ & $1.1 \pm 0.1$ & $1.2 \pm 0.4$ & $1.9 \pm 0.4^{* *}$ \\
$\Delta$ PPTA & $0.5 \pm 0.1$ & $0.3 \pm 0.1$ & $3.6 \pm 0.5^{* *}$ & $2.1 \pm 0.5^{* *}$ \\
Follow up (months) & $2.3 \pm 0.1$ & $2.1 \pm 0.2$ & $2.1 \pm 0.6$ & $2.3 \pm 0.6$ \\
\hline
\end{tabular}

LHF lateral hinge fracture, MPTA medial proximal tibial angle, $P P T A$ posterior proximal tibial angle, $H K A$ hip-knee-ankle angle

**Mean value of the parameter statistically different than no LHF group mean value

\begin{tabular}{lcccc}
\hline Parameters/LHF type & No LHF $(n=84)$ & Type 1 $(n=30)$ & Type 2 $(n=3)$ & Type 3 $(n=3)$ \\
\hline Bone union (months) & $4.3 \pm 0.2$ & $4.3 \pm 0.3$ & $6.7 \pm 1.0^{* *}$ & $9.3 \pm 1.0^{* *}$ \\
Return to work (months) & $2.4 \pm 0.2$ & $2.3 \pm 0.3$ & $4.7 \pm 1.0^{* *}$ & $7.3 \pm 1.0^{* *}$ \\
Return to sports (months) & $5.0 \pm 0.3$ & $4.9 \pm 0.5$ & $5.7 \pm 1.6$ & $13.3 \pm 1.6^{* *}$ \\
$\Delta$ KOOS pain & $31.5 \pm 2.4$ & $31.9 \pm 4.2$ & $33.0 \pm 15.4$ & $20.0 \pm 21.8$ \\
$\Delta$ KOOS symptom & $32.7 \pm 2.4$ & $34.8 \pm 3.9$ & $30.0 \pm 15.2$ & $20.0 \pm 21.5$ \\
$\Delta$ KOOS ADL & $33.1 \pm 2.5$ & $34.8 \pm 4.3$ & $24.0 \pm 15.7$ & $21.0 \pm 22.3$ \\
$\Delta$ KOOS sports & $36.3 \pm 3.2$ & $26.0 \pm 5.0$ & $15.3 \pm 15.2$ & $5.0 \pm 18.6$ \\
$\Delta$ KOOS QOL & $37.8 \pm 3.5$ & $52.2 \pm 6.5$ & $18.0 \pm 21.2$ & $2.5 \pm 21.2$ \\
\hline
\end{tabular}

$K O O S$ knee injury and osteoarthritis outcome score, ADLactivity of daily life, $Q O L$ quality of life, UCLA University of California Los Angeles, $L H F$ lateral hinge fracture

**Mean value of the parameter statistically different than no LHF group mean value
Takeuchi et al. [3] reported a $25 \%$ rate of LHF during OWHTO, diagnosed either intraoperatively or on postoperative radiographs taken just after surgery. Dexel et al. [9] reported an incidence of $30.4 \%$, half of which occurred during the procedure and the other half found on radiographs at the 6 weeks follow-up mark. In this study, the overall LHF rate was $30 \%$, half of these fractures were diagnosed intraoperatively or on immediate postoperative radiographs. It is important to note that a number of studies do not report the specifics of the type of lateral hinge fracture rate occurring during follow up. It is accepted that secondary Takeuchi type 1 fractures found during follow up are almost certainly created at the time of the procedure, but are often not seen with either fluoroscopy or plain X-ray. These subtle fractures are cracks that propagate through the hinge during the process of sawing and distraction; probably would be detected if CT scan was used in immediate postoperative exams [25].

Lee et al. [12] and Munier et al. [2] focused on various cutting guides to achieve better correction and to reduce intraoperative complications during OWHTO. In their comparative study, Lee et al. [13] reported improvement in complications, including lateral hinge fractures using a releaser and a protective cutting complex. They reported superiority of their "protective cutting system" over conventional technique.
Diffo Kaze et al. [15] focused on the effect of hinge drilling to improve the resistance to fracture during OWHTO. Their results confirm that, for tibia vara corrective osteotomies $<5^{\circ}$, drilling a hole at the apex of the osteotomy reduces stresses in the lateral hinge and increases the critical angle before failure of the hinge, but only for corrections inferior to $5^{\circ}$. Similarly, for osteotomies superior to $9 \mathrm{~mm}$ of opening, Bujnowski et al. [14] recently demonstrated, in their ex-vivo study, that a pilot hole provided no significant decrease in the strains experienced at the lateral cortex, nor did it reduce the risk of fracture of the lateral tibial hinge.

Computer-assisted surgery (CAS) can also offer solutions to the surgeon by controlling the depth of the cut, however this alone does not prevent the hinge to be susceptible to fracture [26]. More recently, Dessyn et al. [11] in their comparative cadaveric study using patient-specific cutting guides (PSCG) [2] demonstrated an improvement of the lateral hinges resistance to fracture during osteotomy opening using an additional K-wire intersecting the cutting plane at the theoretical lateral hinge location. These results are supported by Diffo Kaze et al. [15]; in these two studies, angulation, vertical displacement, and maximum load before breakage were all greater when the hinge was protected before applying an opening load. Our study justifies and advocates a 
protective K-wire, which acts as a saw-protector and strain absorber, which protects the hinge during opening.

Using a locked medial plate system during OWHTO, Kim et al. [27] demonstrated in their retrospective study comparing a group without LHF and patients with LHF types I and II did not show any functional deterioration or radiological changes during midterm follow up. Their study confirmed that the occurrence of hinge fractures (Takeuchi types I and II) did not have comparatively worse KOOS subscores at their final follow up. However, patients with Takeuchi II and III fractures returned to their profession and sports activities later than patients with Takeuchi I or no LHF.

We recognize there are some limitations to this study. First, this is a retrospective analysis of data which have been recorded prospectively. As such the retrospective nature of this study might introduce a potential selection bias, however all the procedures in this study were done with a standardized technique in terms of preoperative planning. The surgical technique was carried out by two senior surgeons using the same fixation device and with standardized postoperative rehabilitation. The short-term follow up in this study may have avoided diagnosing late LHF, however previous published series report this complication to be rare after 6 weeks $[3,9]$.

One further potential bias is with the use of graft material placed in the gap during surgery as this may influence postoperative fracture rates. A more rigid graft might absorb strains that would have otherwise been transmitted to the hinge. In a cadaveric study, Scordino et al. [28] demonstrated that injectable calcium phosphate cement improves the initial maximal torsional strength and stiffness of high tibial osteotomy construct. Schwartz [6] treated their patients suffering from isolated medial knee osteoarthritis secondary to genu varum with a minimally invasive OWHTO using injectable calcium phosphate cement. Among their 19 patients, 18 had union of their osteotomy within 2 months. There were no reports of infections in their study, but they did diagnose one type 3 LHF in their noted complications.

In this present study, patients were allowed to go onto full weight-bearing during their rehabilitation period, however this may have increased the number of LHF seen, the fracture rates of this study was similar to that reported in the literature $[3,9]$.

Finally, Lee et al. [29] demonstrated that surgical experience may improve the surgeon's competency in the prevention of lateral hinge fractures, thus highlighting the need to be trained and experienced to fully respect the lateral tibial hinge. The results obtained by the two seniors surgeons involved (performing more than $50 \mathrm{HTO} /$ year) might be different in non-experienced surgeon hands, but the advantages of the protective $\mathrm{K}$-wire might also be more significant for the latter to provide additional radiological landmarks and a rigid sawing safeguard.

\section{Conclusion}

This study demonstrated that, during OWHTO, positioning a $\mathrm{K}$-wire intersecting the cutting plane at the theoretical lateral hinge location reduced the number of intraoperative lateral hinge fractures. OWHTO using a protective K-wire exhibits shorter time to bone union, return to work and/or sport than patients operated on using exactly the same procedure, but without a K-wire.

Author's contribution CJ and FG have made substantial contributions to acquisition of data or analysis and interpretation of data. ME, AS, $\mathrm{KK}, \mathrm{AW}$, and SP have been involved in drafting the manuscript or revising it critically. MO has made substantial contributions to conception and design or acquisition of data or analysis and interpretation of data. Every author has given final approval of the version to be published and agreed to be accountable for all aspects of the work in ensuring that questions related to the accuracy or integrity of any part of the work are appropriately investigated and resolved.

Funding No funding was required for this study.

\section{Compliance with ethical standards}

Conflict of interest Some of the authors disclosed potential conflict of interest.

Ethical approval Local ethics committee approved our study protocol prior to investigation.

\section{References}

1. Flecher X, Parratte S, Aubaniac JM, Argenson JNA (2006) A 12-28-year follow-up study of closing wedge high tibial osteotomy. Clin Orthop 452:91-96

2. Munier M, Donnez M, Ollivier M, Flecher X, Chabrand P, Argenson JN, Parratte S (2017) Can three-dimensional patient-specific cutting guides be used to achieve optimal correction for high tibial osteotomy? Pilot study. Orthop Traumatol Surg Res 103:245-250

3. Takeuchi R, Ishikawa H, Kumagai K, Yamaguchi Y, Chiba N, Akamatsu Y, Saito T (2012) Fractures around the lateral cortical hinge after a medial opening-wedge high tibial osteotomy: a new classification of lateral hinge fracture. Arthrosc J Arthrosc Relat Surg 28:85-94

4. Fujisawa Y, Masuhara K, Shiomi S (1979) The effect of high tibial osteotomy on osteoarthritis of the knee. An arthroscopic study of 54 knee joints. Orthop Clin North Am 10:585-608

5. Hernigou P, Medevielle D, Debeyre J, Goutallier D (1987) Proximal tibial osteotomy for osteoarthritis with varus deformity. A ten to thirteen-year follow-up study. J Bone Joint Surg Am 69:332-354

6. Schwartz C (2018) Minimally invasive opening wedge tibia outpatient osteotomy, using screw-to-plate locking technique and a calcium phosphate cement. Eur J Orthop Surg Traumatol 28:799-809

7. Sprenger TR, Doerzbacher JF (2003) Tibial osteotomy for the treatment of varus gonarthrosis. Survival and failure analysis to twenty-two years. J Bone Joint Surg Am 85-A:469-474

8. Takeuchi R, Umemoto Y, Aratake M, Bito H, Saito I, Kumagai K, Sasaki Y, Akamatsu Y, Ishikawa H, Koshino T, Saito T (2010) 
A mid term comparison of open wedge high tibial osteotomy vs unicompartmental knee arthroplasty for medial compartment osteoarthritis of the knee. J Orthop Surg 5:1-8

9. Dexel J, Fritzsche H, Beyer F, Harman MK, Lützner J (2017) Open-wedge high tibial osteotomy: incidence of lateral cortex fractures and influence of fixation device on osteotomy healing. Knee Surg Sports Traumatol Arthrosc 25:832-837

10. Nakamura R, Komatsu N, Fujita K, Kuroda K, Takahashi M, Omi R, Katsuki Y, Tsuchiya H (2017) Appropriate hinge position for prevention of unstable lateral hinge fracture in open wedge high tibial osteotomy. Bone Jt J 99-B:1313-1318

11. Dessyn E, Sharma A, Donnez M, Chabrand P, Ehlinger M, Argenson J-N, Parratte S, Ollivier M (2019) Adding a protective K-wire during opening high tibial osteotomy increases lateral hinge resistance to fracture. Knee Surg Sports Traumatol Arthrosc. https:// doi.org/10.1007/s00167-019-05404-7

12. Lee YS, Lee MC, Kang SG, Elazab A, Oh WS (2016) Openwedge high tibial osteotomy using a protective cutting system: technical advancement for the accuracy of the osteotomy and avoiding intraoperative complications. Arthrosc Tech 5:e7-e10

13. Lee YS, Moon GH (2015) Comparative analysis of osteotomy accuracy between the conventional and devised technique using a protective cutting system in medial open-wedge high tibial osteotomy. J Orthop Sci 20:129-136

14. Bujnowski K, Getgood A, Leitch K, Farr J, Dunning C, Burkhart TA (2018) A pilot hole does not reduce the strains or risk of fracture to the lateral cortex during and following a medial opening wedge high tibial osteotomy in cadaveric specimens. Bone Jt Res 7:166-172

15. Kaze AD, Maas S, Hoffmann A, Pape D (2017) Mechanical strength assessment of a drilled hole in the contralateral cortex at the end of the open wedge for high tibial osteotomy. J Exp Orthop 4(1):23

16. Jacquet C, Sharma A, Fabre M, Ehlinger M, Argenson J-N, Parratte S, Ollivier M (2019) Patient-specific high-tibial osteotomy's "cutting-guides" decrease operating time and the number of fluoroscopic images taken after a Brief Learning Curve. Knee Surg Sports Traumatol Arthrosc. https://doi.org/10.1007/s00167-01905637-6

17. Ahlbäck S (1968) Osteoarthrosis of the knee. A radiographic investigation. Acta Radiol Diagn Suppl 277:7-72

18. Kellgren JH, Lawrence JS (1957) Radiological assessment of osteo-arthrosis. Ann Rheum Dis BMJ Publ Group 16:494-502

19. Elson DW, Petheram TG, Dawson MJ (2015) High reliability in digital planning of medial opening wedge high tibial osteotomy, using Miniaci's method. Knee Surg Sports Traumatol Arthrosc 23:2041-2048
20. Ollivier M, Bulaïd Y, Jacquet C, Pesenti S, Argenson NJ, Parratte S (2018) Fixation augmentation using calcium-phosphate bone substitute improves outcomes of complex tibial plateau fractures. A matched, cohort study. Int Orthop 42:2915-2923

21. Ornetti P, Parratte S, Gossec L, Tavernier C, Argenson J-N, Roos EM, Guillemin F, Maillefert JF (2008) Cross-cultural adaptation and validation of the French version of the Knee injury and Osteoarthritis Outcome Score (KOOS) in knee osteoarthritis patients. Osteoarthritis Cartilage 16:423-428

22. Zahiri CA, Schmalzried TP, Szuszczewicz ES, Amstutz HC (1998) Assessing activity in joint replacement patients. J Arthroplasty 13:890-895

23. Jo H-S, Park J-S, Byun J-H, Lee Y-B, Choi Y-L, Cho S-H, Moon D-K, Lee S-H, Hwang S-C (2018) The effects of different hinge positions on posterior tibial slope in medial open-wedge high tibial osteotomy. Knee Surg Sports Traumatol Arthrosc 26:1851-1858

24. Varga P, Zysset PK, Schefzig P, Unger E, Mayr W, Erhart J (2016) A finite element analysis of two novel screw designs for scaphoid waist fractures. Med Eng Phys 38:131-139

25. Lee OS, Lee YS (2018) Diagnostic value of computed tomography and risk factors for lateral hinge fracture in the open wedge high tibial osteotomy. Arthrosc J Arthrosc Relat Surg 34:1032-1043

26. Saragaglia D, Chedal-Bornu B, Rouchy RC, Rubens-Duval B, Mader R, Pailhé R (2016) Role of computer-assisted surgery in osteotomies around the knee. Knee Surg Sports Traumatol Arthrosc 24:3387-3395

27. Il KK, Kim GB, Kim HJ, Lee SH, Yoon WK (2018) Extra-articular lateral hinge fracture does not affect the outcomes in medial open-wedge high tibial osteotomy using a locked plate system. Arthrosc J Arthrosc Relat Surg 34:3246-3255

28. Scordino LE, Obopilwe E, Charette R, Edgar CM, DeBerardino TM, Mazzocca AD (2017) Calcium phosphate cement enhances the torsional strength and stiffness of high tibial osteotomies. Knee Surg Sports Traumatol Arthrosc 25:817-822

29. Lee DK, Kim KK, Ham CU, Yun ST, Kim BK, Oh KJ (2018) The learning curve for biplane medial open wedge high tibial osteotomy in 100 consecutive cases assessed using the cumulative summation method. Knee Surg Relat Res 30:303-310 